\title{
ORIGINALS
}

\section{Sugar Transport in the Small Intestine of Obese Hyperglycemic, Fed and Fasted Mice}

\author{
I. Bihler and N. Freund \\ Dept. of Biochemistry, Imperial College of Science and Technology, London, England
}

Received: December 3, 1974, and in revised form: May 26, 1975

\begin{abstract}
Summary. 1 . The in vitro transport of 3-0-methyl-D-glucose was measured in the small intestine of obese-hyperglycemic (ob/ob) mice and their lean littermates, fed or fasted for $48 \mathrm{hrs}$. 2. Transport was much increased in the jejunum of obese animals and, to a lesser extent, in obese mice on a chronic restricted diet. 3. Kinetic studies indicate that the Vmax of transport was significantly greater in obese than in lean mice, whether fed or fasting. Fasting increased the Vmax in lean but not in obese animals. These changes were more prominent in the jejunum. The apparent $\mathrm{Km}$ of transport was
\end{abstract}

the same in all four groups. 4. These findings are discussed in relation to the increase in intestinal absorptive functions in diabetes and in some conditions of food restriction or starvation. The results are consistent with the hypothesis that the effects of diabetes and of starvation on intestinal sugar transport reflect an alteration in the same controlling factor.

Key words: ob/ob mice, intestinal sugar transport, kinetics, diabetes, fasting.
It is by now well established that the intestinal absorption of glucose and related sugars is increased in diabetes [1-11]. Although numerous recent studies have dealt with this effect, its nature is at present little understood. It would appear, however, that this effect does not involve an immediate alteration in the activity of the sugar transport system, but rather an adaptive change which requires several days to develop or to be reversed.

Restriction of food intake and complete starvation have also been shown to significantly alter sugar absorption in the small intestine [9-18]. These effects are highly complex, with stimulation or inhibition predominating, depending on several factors, including the duration of the fast and the nature of the sugar being transported. Under some experimental conditions sugar transport is definitely increased, and this effect also appears to develop gradually, as that in diabetes. To our knowledge, the effects of fasting and of diabetes on the kinetics of intestinal sugar absorption have not been compared directly nor have they been investigated when occurring simultaneously.

In this investigation the obese-hyperglycemic mouse (ob/ob) was used as a model for experimental diabetes. Intestinal sugar transport in vitro was studied in obese mice and their lean littermates in the fed and fasting state. Kinetic parameters of the transport system were determined in each case.

\section{Materials and Methods}

The obese (ob/ob) mice and their lean littermates were derived from the obese-hyperglycemic mouse colony originated at the Jackson Memorial Laboratory, Bar Harbor, Maine, U.S. A., as described in a previous publication [19]. The animals studied were 2-3 month old males; at this age obesity was already well developed (see Table 1) and lean animals had nearly reached stable weight [19]. The "fed" animals had free access to food and water, whereas the "fasted" animals were deprived of food for $48 \mathrm{hrs}$ before sacrifice. Obese animals on a restricted diet (ob/ob RD) were treated as follows. They were separated from their littermates as soon as obesity could be indisputably detected (approx. 28 to 35 days) and given about $3.7 \mathrm{~g} /$ day standard mouse diet when 5 weeks old, about $4.4 \mathrm{~g} /$ day when six weeks old and $5-6 \mathrm{~g} /$ day from the seventh week onwards. This diet was sufficient to ensure normal growth but maintained their body weight only slightly above that of comparable lean animals. These obese restricted diet mice were used at 6-12 month of age.

Intestinal sugar transport was measured in transversely cut everted segments of small intestine prepared according to Crane and Mandelstam [20]. The incubation flasks contained a number of segments (150 to $200 \mathrm{mg}$ total weight) representative of either the whole small intestine or of the jejunum or ileum 
separately. The proximal $2 / 5$ of the small gut were taken as jejunum. The tissue was incubated with gentle shaking at $37^{\circ}$ in $4.0 \mathrm{ml}$ Krebs-Henseleit [21] bicarbonate buffer, $\mathrm{pH} 7.4$, equilibrated with $95 \%$ $\mathrm{O}_{2}-5 \% \mathrm{CO}_{2}$. The incubation medium contained a mixture of ${ }^{14} \mathrm{C}$-labelled and unlabelled 3-0methyl-D-glucose at the concentration indicated and tracer amounts of ${ }^{3} \mathrm{H}$-labelled inulin, serving as extracellular marker. The tissues were incubated for $15 \mathrm{~min}$ and then removed from the medium, gently blotted, weighed on a torsion balance and extracted with water in a boiling water bath for $10 \mathrm{~min}$. The tissue extract and samples of the incubation medium were deproteinized with trichloroacetic acid ( $5 \%$ final concentration) and the radioactivity was measured by double label liquid scintillation spectrometry. The concentration of 3-methylglucose in the intracellular tissue water space was calculated by applying a correction for total tissue water content, determined in separate experiments, and for the inulin space determined in each sample. Results are expressed in terms of percent filling, i.e., the concentration in the intracellular water is given as percent of the concentration in the medium. Statistical evaluation of the data in Tables 1 and 2 was done by Student's t-test and of the data in Figs. 2 and 3 by analysis of variance in a $2 \times 2$ factorial design, applied to the results of computations of kinetic constants by the procedures of Bliss and James [22].

The radiosotopes used were from the Radiochemical Centre, Amersham, England: 3-0methyl-[D-glucose- $\left.{ }^{14} \mathrm{C}(\mathrm{U})\right]$, sp. act. $24 \mathrm{mCi} / \mathrm{mmol}$, and inulin- ${ }^{3} \mathrm{H}$ (generally labelled), sp. act. 300 $\mathrm{mCi} / \mathrm{mmol}$. The latter had a radiochemical purity of $>98 \%$ and was fractionated by gel filtration to yield a homogeneous product with a M. W. distribution identical to that of native inulin. Hence, it did not contain low molecular weight components which were occasionally found in methoxy- ${ }^{3} \mathrm{H}$-inulin and in carboxyl- ${ }^{14} \mathrm{C}$-inulin of some other suppliers [23, 24]. Other chemicals were analytical grade from a variety of sources.

\section{Results}

Data on body weight and intestinal size of ob/ob mice and their lean littermates are summarized in Table 1 . Gut weight and gut length in the obese mice were significantly increased but to a proportionately lesser extent than total body weight. Fasting for $48 \mathrm{hrs}$ resulted in a significant decrease in body weight of both lean and obese mice but the decrease in gut weight was significant in the obese animals only. Gut length was not significantly changed by fasting. The gut weight/length ratio was significantly higher in the fed ob/ob mice than in fasted ob/ob mice or in lean animals, whether fed or fasted.

In this study the in vitro accumulation within the tissue of the non-metabolized sugar 3-methylglucose is taken as a measure of activity of the sugar transport system and is expressed as a "filling" or accumulation ratio. Preliminary experiments (not shown) indicated that the transport rate remained constant for at least $20 \mathrm{~min}$; sugar accumulation during the $15 \mathrm{~min}$ incubation period is, therefore, an adequate measure of the initial inward transport rate.

Table 2 shows the results from experiments with a single low $(0.5 \mathrm{mM})$ concentration of 3-methylglucose and compares results obtained in the jejunum and ileum of lean, ob/ob and ob/ob RD animals. The data indicate that active accumulation of 3 -methylglucose is taking place, with $3-5$ fold accumulation of the sugar in the tissue occurring within the $15 \mathrm{~min}$ incubation period. The results clearly show that sugar transport is significantly increased in the jejunum of obese animals and, to a lesser extent but still significantly, in the jejunum of obese animals kept on a restricted diet.

Table 1. Body weight and intestinal size

\begin{tabular}{llclll}
\hline & \multicolumn{2}{l}{ lean } & & \multicolumn{2}{l}{ ob/ob } \\
\cline { 2 - 3 } \cline { 5 - 6 } & fed & fasted & & fed & fasted \\
\hline body weight, g & $34.3 \pm 1.1(17)$ & $27.3 \pm 1.4(7)^{\mathrm{a}}$ & & $57.6 \pm 0.9(15)^{\mathrm{b}}$ & $51.9 \pm 1.5(8)^{\mathrm{a}, \mathrm{b}}$ \\
gut weight, $\mathrm{g}$ & $1.97 \pm 0.84(15)$ & $1.78 \pm 0.05(8)$ & & $2.74 \pm 0,07(15)^{\mathrm{b}}$ & $2.21 \pm 0.09(8)^{\mathrm{a}, \mathrm{b}}$ \\
gut length, cm & $47.2 \pm 0.8(24)$ & & \multicolumn{2}{c}{$57.9 \pm 1.0(25)^{\mathrm{b}}$} \\
gut wt./gut length, $\mathrm{mg} / \mathrm{cm}$ & $39.6 \pm 1.7(14)$ & $37.6 \pm 3.2(7)$ & & $47.9 \pm 2.0(15)^{\mathrm{c}}$ & $38.7 \pm 0.9(8)^{\mathrm{a}}$ \\
gut wt./body wt., $\mathrm{mg} / \mathrm{g}$ & 57.4 & 65.9 & & 47.6 & 42.6 \\
\hline
\end{tabular}

\footnotetext{
a $p<0.005$ compared to corresponding fed.

${ }^{b} p<0.001$ compared to corresponding lean.

${ }^{c} p<0.005$ compared to corresponding lean.
} 
Table 2. Transport of $0.5 \mathrm{mM} 3$-methylglucose

\begin{tabular}{lll}
\hline \multicolumn{3}{l}{$\%$ intracellular filling } \\
\cline { 2 - 3 } & jejunum & ileum \\
\hline Lean & $283.6 \pm 20.7(12)$ & $311.1 \pm 36.4(10)$ \\
ob/ob & $492.5 \pm 57.6(11)^{\mathrm{a}}$ & $354.1 \pm 26.8(10)^{\mathrm{c}}$ \\
ob/ob RD & $375.6 \pm 30.0(10)^{\mathrm{b}}$ & $305.9 \pm 37.9(7)$
\end{tabular}

a $P<0.005$ compared to lean jejunum.

b $P<0.05$ compared to lean jejunum and to ob/ob jejunum.

c $P<0.05$ compared to ob/ob jejunum.

Experimental details are given in the text. Values are concentrations in intracellular water space expressed as percent of the final concentration in the incubation medium (mean \pm S.E.M.), with the number of determinations in parentheses.

Transport in the ileum was not significantly different in the three groups although a trend towards increased transport was seen in the obese animals. There was no significant difference between transport rates in jejunum and ileum of lean and ob/ob RD animals but the difference between the two parts of the small intestine was significant in the obese group. The value of this experiment is limited in that the only available ob/ob RD animals, although not much heavier (mean weight \pm S.E., $42.0 \pm 2.1 \mathrm{~g}$ ), were considerably older than those in the other two groups; it is also well known [25] that comparisons of transport rates at one arbitrarily chosen concentration may show a misleading picture.

To obtain more detailed information on the nature of the differences in sugar transport associated with the ob/ob genetic trait and with fasting, a number of kinetic experiments were performed. In each experiment five or more sugar concentrations ranging from 5-33 $\mathrm{mM}$ were tested in triplicate, using separately pooled tissues from three obese and three lean animals. Two such experiments were done with jejunal and two with ileal tissues of fed animals, and the same experimental design was followed with fasted animals. Thus, lean and obese littermates were compared in the same experiment but comparisons between the fed and fasted state, or between jejunum and ileum necessarily involve different groups of animals. However, animals in all experiments were of matched age, so that there was very little variation in weight within each of the four catagories (see Table 1) and presumably, within the lean or obese groups if compared before fasting.

The results obtained in these experiments were treated according to the procedure of Bliss and James [22], in which the data are fitted to a hyperbola by the procedure of maximum likelihood. This computerized procedure is not subject to personal bias in curve fitting nor does it give exaggerated statistical weight to the points obtained at lowest substrate concentrations, both of which are recognized shortcomings of the familiar Lineweaver-Burke [26] double reciprocal treatment. The values for $\mathrm{Km}$ and Vmax obtained in this manner thus represent unbiased estimates for the kinetic constants (and their standard error), assuming that the transport process obeys Michaelis-Menten kinetics. This assumption has been repeatedly verified in many experimental systems. To illustrate the nature of the data obtained Fig. 1 shows a conventional Lineweaver-Burke plot of the data from jejunum. The points are experimental means ( \pm S.E.), and the regression lines are constructed from the parameters calculated by the Bliss and James procedure. The agreement is very good.

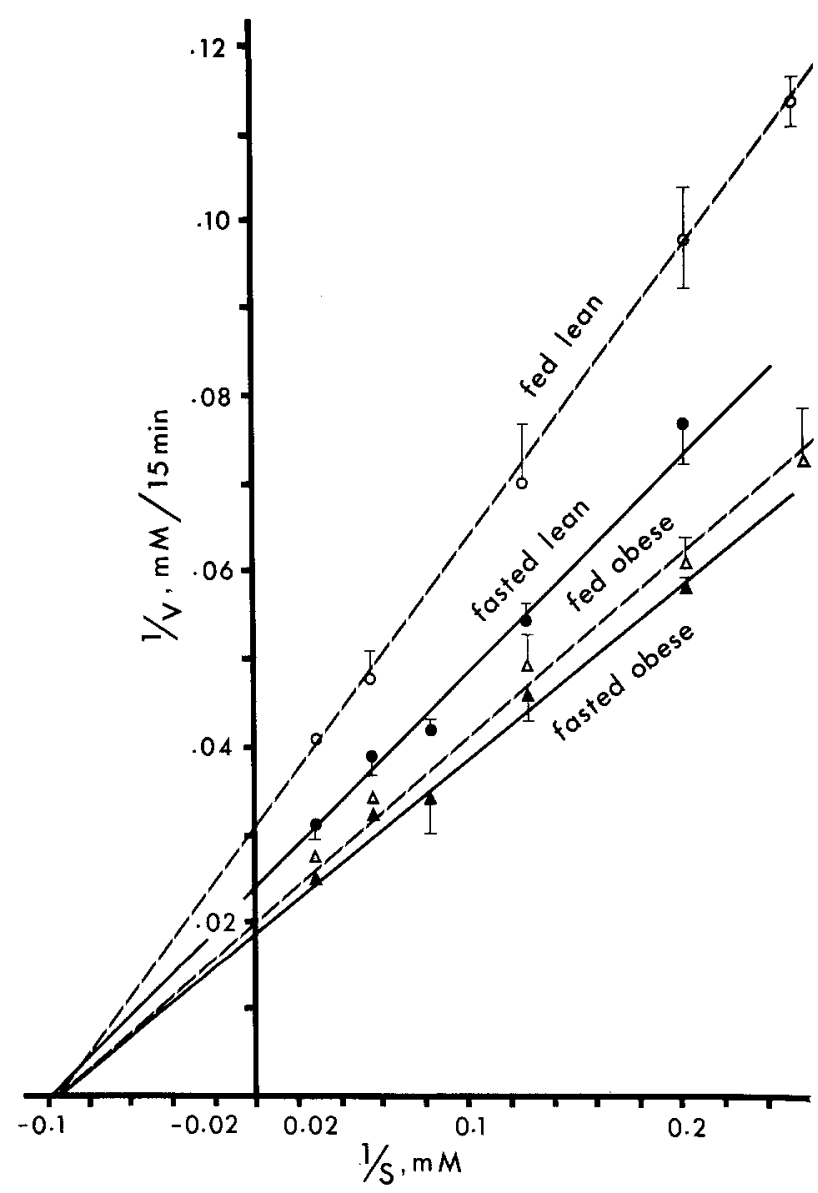

Fig. 1. Kinetics of 3-methylglucose transport in jejunum of fasted $\mathrm{ob} / \mathrm{ob}$ and lean mice. Lineweaver-Burke double reciprocal plot constructed from data treated according to Bliss and James [22]. For details see text 


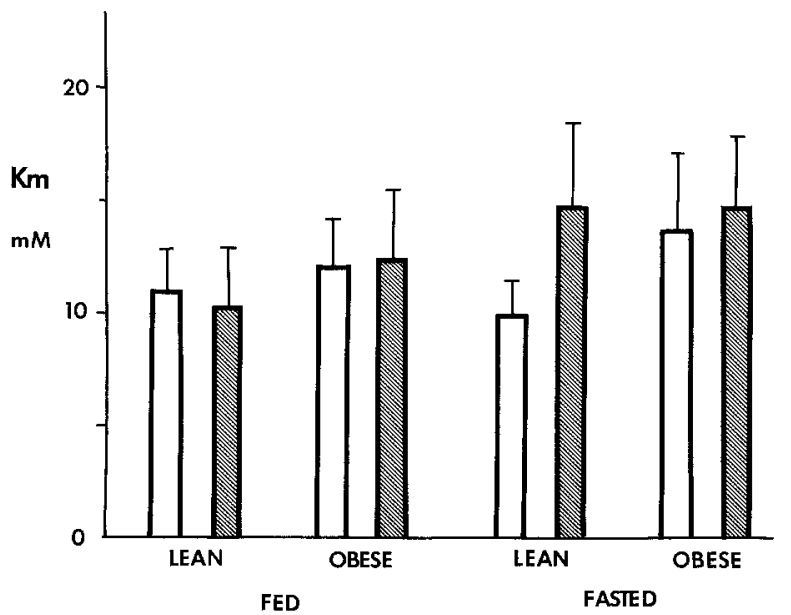

Fig. 2. Km of 3-methylglucose transport in fed and fasted, lean and ob/ob mice. The bars indicate means ( \pm S.E.M.) calculated by the method of Bliss and James [22] from two experiments in triplicate, each at 5 different sugar concentrations, ranging from 5 to $33 \mathrm{mM}$. For detail see text. Hollow bars indicate jejunum and hatched bars ileum

A complete summary of results for jejunum and ileum of fed and fasted, obese and lean mice is given in Fig. 2 for the apparent $\mathrm{Km}$ values and in Fig. 3 for the corresponding Vmax values. Analysis of variance indicates there were no significant differences in the $\mathrm{Km}$ values of sugar transport between any of the groups. In contrast, there were highly significant changes in the Vmax. In jejunum, the Vmax of the fed obese, fasted obese and fasted lean groups were all significantly higher than that of the fed lean goup. The Vmax of the fasted obese group was also significantly higher than of the fasted lean group. In other words, obesity increased Vmax in fed $(p<0.01)$ and fasted $(p$ $<0.05)$ mice, whereas fasting was effective in lean $(p$ $<0.01$ ) but not in obese animals. In the ileum, the effects were less pronounced: Obesity increased $V \max$ in fed $(p<0.05)$ but not in fasted animals; fasting did not cause a significant change in either lean or obese mice, but the effect of obesity and fasting combined was significant at a higher level $(p<0.01)$ when compared to the fed lean group. Thus, sugar transport in the obese animals has a higher capacity than in the lean ones and, in the jejunum, fasting for $48 \mathrm{hrs}$ evokes a similar increase in the maximal capacity of the sugar transport system. As for the interaction between the ob/ob effect and fasting, the data indicate that the genetic effect seems to add to the effect of fasting but that fasting of the obese animals does not elicit any additional increase in transport capacity. Within each of the four categories there was no significant difference between transport capacities in the jejunum and ileum.

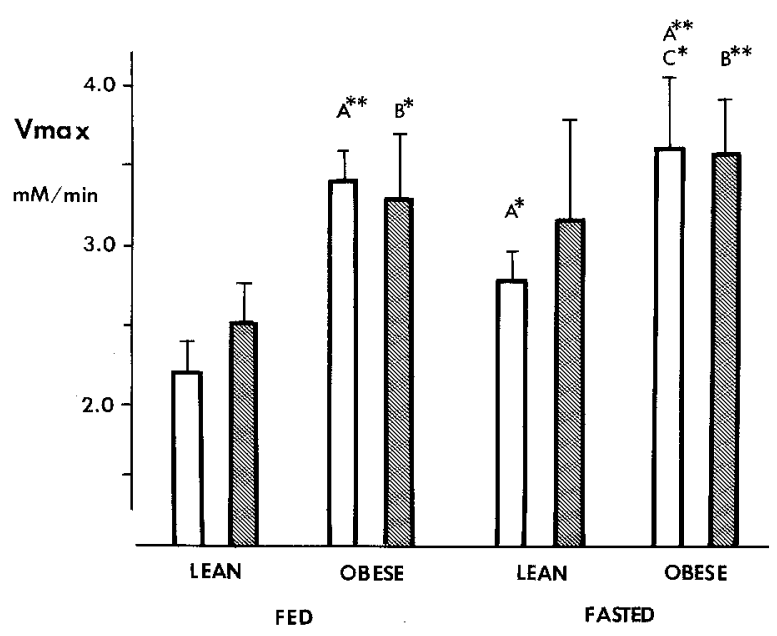

Fig. 3. Vmax of 3-methylglucose transport in fed and fasted, lean and ob/ob mice. For details see text and Fig. 2. The symbols above the bars indicate significance of difference compared to, $\mathrm{A}$-fed lean jejunum, B - fed lean ileum, $\mathrm{C}-$ fasted lean jejunum

$* p<0.05$

$* * p<0.01$

\section{Discussion}

The obese hyperglycemic mouse (ob/ob) is characterized by gross obesity, hyperglycemia, hyperinsulinemia and other hormonal and metabolic changes strikingly resembling the situation in human adult-onset diabetes mellitus [27]. For the study of intestinal sugar transport the ob/ob mouse has several advantages, among them that diabetes has been established for a considerable time, is not induced by potentially toxic agents, such as alloxan, and is not being treated with insulin. It also permits comparison between obese animals and their lean, heterozygous littermates and studies on the effect of starvation and dietary restriction.

Intestinal absorption in ob/ob mice has been studied before. Mayer and Yannoni [28] have found that glucose absorption was increased in the ob/ob mice and that this was not related to alterations in gastric emptying or to hyperglycemia. They concluded that increased carbohydrate absorption in these animals is an adaptive phenomenon related to hyperphagia but this is not supported by their observation that chronic underfeeding of ob/ob mice did not cause a significant decrease in glucose absorption. More recently, Grimmel et al. [29] reported that the activity of several disaccharidases was increased in $\mathrm{ob} / \mathrm{ob}$ mice but that galactose transport in vitro was not significantly greater than in the lean littermates. The galactose concentration used in their study, $\mathbf{5 5 . 5}$ $\mathrm{mM}$, was so high, however, that any changes in the specific active sugar transport system could have been 
overshadowed by large diffusional fluxes, osmotic changes, etc.

Relatively low concentrations of the nonmetabolized glucose analog 3-methylglucose were used in the present study. Recent work has provided evidence for the existence of multiple sugar transport systems in the intestine $[16,30]$. Although 3-methylglucose appears not to be a substrate for all these systems it was used here because it reflects the activity of the quantitatively most important sodiumdependent active transport system responsible for the absorption of free glucose. Since the absorptive surface area cannot be determined directly, the data are expressed as relative sugar concentrations in the intracellular water space. This parameter depends also on rates of sugar exit from the tissue and on the size of the compartment available for sugar accumulation. However, the effect of sugar exit is minimal because accumulation during the 15 min incubation period closely approximates initial entry rates. On the other hand, the smaller size of the aqueous compartment in the thinner ileal tissue makes direct comparison with the thicker jejunal tissue more difficult. It has been shown, for example, that the increase in galactose transfer across the gut wall after fasting is accompanied by only small alterations in ileal galactose content [18]. This might explain why we found only small or insignificant effects in the ileum. In view of the indirect relationship between tissue accumulation and transporting activity of the absorptive surface, it should be pointed out that the results are directly comparable in the three goups of Table 1 where the gut weight/length ratio is the same, but the true absorptive rates in the obese fed group could be underestimated.

The data in Table 2 compare the transport of 0.5 $\mathrm{mM}$ 3-methylglucose in lean and ob/ob mice and in $\mathrm{ob} / \mathrm{ob}$ mice maintained chronically on a restricted diet. They indicate that transport in the jejunum was significantly increased in the obese animals. This effect was partially but not completely reversed by dietary restriction, in line with observations that dietary restriction does not completely reverse a variety of other metabolic abnormalities in the ob/ob genetic trait [19]. Although the ob/ob RD animals were older (6-12 months) than the ob/ob and lean animals (2-3 months) they had reached a stable weight [19], which was only slightly above that of the lean animals. Therefore, a rough comparison with the other two groups was still possible.

The kinetic experiments summarized in Figs. 2 and 3 indicate that the increase in sugar transport in the obese animals is due to a greater maximal capacity of the transport system rather than to a change in the system's affinity for sugar, expressed by the apparent $\mathrm{Km}$. The increase in maximal transport capacity shown here is in agreement with the data of Olsen and Rosenberg [7] and of Caspary et al. [8], obtained with animals made diabetic with alloxan or streptozotocin.

Fasting for $48 \mathrm{~h}$ induced a similar change in transport parameters, but the increase in Vmax was seen only in lean animals and was of smaller magnitude than that associated with the genetic defect. Recent studies $[18,31]$ have helped to resolve some earlier contradictions in the effect of fasting. Sanford and Smyth [18] have shown that starvation definitely increases intestinal sugar transport but, at the same time, decreases the availability of hexose-derived energy to the transfer mechanism. Also, the relative contribution of hexose metabolism to transport varies along the length of the small intestine. These facts explain how fasting may either enhance or depress intestinal sugar absorption, depending on whether the energy supply is rate limiting. Furthermore, Schanbacher et al. [31] recently reported that the absorption of glucose was unchanged, but its metabolism by the intestinal tissue was greatly diminished, in rats maintained by isocaloric intravenous alimentation, illustrating the difference between the effect of true starvation and of absence of glucose from the lumen.

We have attempted to minimize the effect of these variables by measuring the transport of a nonmetabolized sugar in vitro. In agreement with Sanford and Smyth [18] and others, our data show that starvation increases sugar transport. This was best seen in the jejunum of lean animals but was only minor in the ileum; it was apparently not additive to the similar increase linked to the genetic defect. In contrast, our data showing that this increase in sugar transport upon fasting is associated with an increase in Vmax do not agree with the recent report of Debnam and Levin [17] who derived kinetic constants from in vitro measurements of transmural electrical potentials and concluded that both $\mathrm{Km}$ and Vmax are decreased in starvation.

It is well established that there is a time lag of several days between the induction of diabetes and the increase in sugar transport. At the same time, a number of other changes in intestinal function become apparent, such as an increase in the transport of amino acids [32], bile acids [33] and sodium [11], as well as in the activity of the brush border disaccharidases, maltase, isomaltase and sucrase [29,34-37]. There is also a similar lag between the administration of insulin in vivo and the reversal of this effect. The time delay and the multiplicity of changes in intestinal function 
suggest that the effects may be associated with the appearance of a new generation of intestinal epithelial cells, rather than with a short term alteration in the activity of transport systems and enzymes. If such changes are associated with an increase in the amount of carrier or enzyme present, this would be reflected in an increased Vmax of the system.

Several explanations have been put forward to explain this effect, among them: 1 . Hyperphagia and intestinal hypertrophy; this is not consistent with observations that these phenomena appear much later than the increase in intestinal transport when diabetes is induced chemically [38], nor with the persistence of increased transport in ob/ob mice on a restricted diet [28] and Table 2. 2. Increased steroid levels; mineralocorticoid administration increases intestinal sugar absorption [4], but there is no evidence that the level of these hormones is increased in all stages of diabetes or starvation. 3. Decreased protein synthesis, as shown in model experiments with cycloheximide [39]; these data might explain slower intestinal cell growth and decreased disaccharidase activities in starvation but they do not explain how a generalized decrease in the synthesis of cellular constituents during starvation or diabetes could increase intestinal absorptive functions. 4. Increase in the glucagon/in sulin ratio; Rudo and Rosenberg [40] have shown that chronic glucagon administration increases sugar and amino acid transport in the gut and have pointed out the possible significance of this effect in view of the demonstrated increase in glucagon/insulin ratios during food restriction and starvation [41] and in diabetes [42]. Present evidence, or rather the lack of it, does not exclude other mechanisms, however.

This study does not provide a final answer but it shows a similarity in the stimulatory effects of diabetes and starvation on intestinal sugar transport: This process is affected in the same manner by the metabolic changes stemming from the ob/ob genetic trait and from fasting. These observations are compatible with the hypothesis [40] that the same factor, namely a change in the glucagon/insulin ratio, is responsible for the effect in both instances. This hypothesis is made attractive by the fact that starvation and diabetes cause very similar changes in many other metabolic variables as well.

Acknowledgements. We thank Professor Sir Ernst Chain for providing laboratory space and other support and Drs. Anne Beloff-Chain and David J. Hearse for advice and encouragement. This work was supported by a grant from the Medical Research Council of Canada. I.B. is a Research Associate of the Medical Research Council of Canada and thanks the Council for support of extended travel leave during the term of this investigation.

\section{References}

1. Laszt, L., Vogel, H.: Resorption of glucose from the small intestine of alloxan - diabetic rats. Nature (Lond.) 157 , 551-552 (1946)

2. Crane, R.K.: An effect of alloxan - diabetes on the active transport of sugars by rat small intestine, in vitro. Biochem. biophys. Res. Commun. 4, 436-440 (1961)

3. Vinnik, I.E., Kern, F., Jr., Sussman, K.E.: The effect of diabetes mellitus and insulin on glucose absorption by the small intestine in man. J. Lab. clin. Med. 66, 131-136 (1965)

4. Müller, F., Beyreiss, K., Dettmer, D., Hartenstein, H.: Untersuchungen zur Wirkung des Alloxandiabetes auf die aktive Resorption von Monosacchariden. Acta biol. med. germ. 19, 673-681 (1967)

5. Leese, H.J., Mansford, K.R.L.: Effect of insulin deficiency on the transport of glucose by rat small intestine. FEBS Letters 2 , 193-194 (1969)

6. Flores, P., Schedl, H.P.: Intestinal transport of 3-0-methyl-D-glucose in the normal and alloxan - diabetic rat. Amer. J. Physiol. 214, 725-729 (1968)

7. Olsen, W.A., Rosenberg, I.H.: Intestinal transport of sugars and amino acids in diabetic rats. J. clin. Invest. 49, 96-105 (1970)

8. Caspary, W.F., Rhein, A.M., Creutzfeldt, W.: Increase of digestive and transport functions of intestinal mucosa from streptozotocin - diabetic rats. Europ. J. clin. Invest. 2, 278 (1972)

9. Axelrad, A. D., Lawrence, A. L., Hazelwood, R. L.: Fasting and alloxan diabetes effects on intestinal transport of monosaccharides. Amer. J. Physiol. 219, 860-864 (1970)

10. Leese, H.J., Mansford, K. R.L.: The effect of insulin and insulin deficiency on the transport and metabolism of glucose by rat small intestine. J. Physiol. (Lond.) 212, 819-838 (1971)

11. Teale, J.D., Love, A.H.G.: Effect of glibenclamide on sugar transport by fed, starved and diabetic rat small intestine. Biochem. Pharmacol. 22, 997-1014 (1973)

12. Kershaw, T.G., Neame, K.D., Wiseman, G.: The effect of semi-starvation on absorption by the rat small intestine in vitro and in vivo. J. Physiol. (Lond.) 152, 182-190 (1960)

13. Wiseman, G.: Effect of undernutrition on intestinal active transport of sugars and amino acids. In: Nutricia Symposium Metabolic processes in the foetus and newborn infant (eds. J.H.P. Jonxis, H.K. A. Visser, H. A. Troelstra) pp. 243-267. Leiden: H. E. Stenfert Kroese N. V. 1971

14. Esposito, G.: Intestinal absorption of sugars in semi-starved rats. Proc. Soc. exp. Biol. (N.Y.) 125, 452-455 (1967)

15. Levin, R.J., Syme, G.: Differential changes in the "apparent $\mathrm{Km}$ " and maximum potential difference of hexose and amino acid electrogenic transfer mechanisms of the small intestine, induced by fasting and hypothyroidism. J. Physiol. (Lond.) 213, 46-48P (1970)

16. Debnam, E. S., Levin, R. J.: Evidence for separate hexose carriers in the small intestine uncovered by fasting and glucose alimentation. J. Physiol. (Lond.) 218, 38-39P (1971)

17. Debnam, E.S., Levin, R.J.: Assessment of the effects of starvation and of semi-starvation on the operational kinetic parameters of the active transfer of hexoses measured in vivo. J. Physiol. (Lond.) 231, 21-23P (1973)

18. Sanford, P.A., Smyth, D.H.: The effect of fasting on hexose transfer in rat intestine. J. Physiol. (Lond.) 239, 285-299 (1973)

19. Abraham, R.R., Beloff-Chain, A.: Hormonal control of intermediary metabolism in obese hyperglycemic mice. I. The sensitivity and response to insulin in adipose tissue and muscle in vitro. Diabetes 20, 522-534 (1971) 
20. Crane, R. K., Mandelstam, P.: The active transport of sugars by various preparations of hamster intestine. Biochim. biophys. Acta (Amst.) 45, 460-476 (1960)

21. Krebs, H.A., Henseleit, K.: Untersuchungen über die Harnstoffbildung im Tierkörper. Hoppe-Seylers Z. physiol, Chem. 210, 33-66 (1932)

22. Bliss, C.I., James, A.T.: Fitting the rectangular hyperbola. Biometrics 22, 573-602 (1966)

23. Esposito, G., Csáky, T.Z.: Extracellular space in the epithelium of rat's small intestine. Amer. J. Physiol. 226, 50-55 (1974)

24. Levi, G.: Different estimates of tissue extracellular space using [Carboxyl- $\left.{ }^{14} \mathrm{C}\right]-$ inulin from different sources. Anal. Biochem. 32, 348-353 (1969)

25. Neame, K.D., Richards, T.G.: Elementary kinetics of membrane carrier transport. p. 27. Blackwell, London: 1972

26. Lineweaver, H., Burke, D.: Determination of enzyme dissociation constants. J. Amer. chem. Soc. 56, 658-666 (1934)

27. Bray. G.A., York, D.A.: Genetically transmitted obesity in rodents. Physiol. Rev. 51, 598-646 (1971)

28. Mayer, J., Yannoni, C.Z.: Intestinal absorption of glucose in three forms of obesity in the mouse. Amer. J. Physiol. 185, 49-53 (1956)

29. Grimmel, K., Rakow, L., Rommel, K., Lacher, K., Burkhardt, F.: Disaccharidasenaktivität und Monosaccharidabsorption bei genetisch adipösen Mäusen. Z. klin. Chem. 8, 570-571 (1970)

30. Honegger, P., Semenza, G.: Multiplicity of carriers for free glucalogues in hamster small intestine. Biochim, biophys. Acta (Amst.) 318, 390-410 (1973)

31. Schanbacher, L.M., Copeland, E.M., Dudrick, S.J., Johnson, L.R., Castro, G.A.: Glucose transport across the small intestine of parenterally nourished rats. Fed. Proc. 34, 917 (1975)

32. Younoszai, M. K., Schedl, H.P.: Intestinal amino acid transport and tissue concentrations in diabetes. Amer. J. Physiol. 223, 828-831 (1972)

33. Caspary, W.F.: Increase of active transport of conjugated bile salts in streptozotocin - diabetic rat small intestine. Gut 14, 949-955 (1973)
34. Olsen, W.A., Rogers, L.: Jejunal sucrase activity in diabetic rats. J. Lab. clin. Med. 77, 838-842 (1971)

35. Younoszai, M.K., Schedl, H.P.: Effect of diabetes on intestinal disaccharidase activities. J. Lab. clin. Med. 79, 579-586 (1972)

36. Rommel, K., Böhmer, R., Goberna, R., Fussgänger, R.: Influence of insulin and glybenclamid on the intestinal disaccharidases of subtotally pancreatectomized rats. Digestion 6, 146-151 (1972)

37. Sharma, A.K., Ghosh, A.K.: Some observations on the intestinal oligosaccharidase activities in normal and alloxan diabetes before and after insulin therapy. Jap. J. exp. Med. 43, 443-446 (1973)

38. Schedl, H.P., Wilson, H.D.: Effect of diabetes on intestinal growth and hexose transport in the rat. Amer. J. Physiol. 220, 1739-1745 (1971)

39. Franklin, J.L., Rosenberg, I.H.: Cycloheximide reproduces diabetic effects on intestinal transport: An approach to the analysis of transport control mechanisms. Gastroenterology 60, 774 (1971)

40. Rudo, N.D., Rosenberg, I. H.: Chronic glucagon adminstration enhances interestinal transport in the rat. Proc. Soc. exp. Biol. (N. Y.) 142, 521-525 (1973)

41. Marliss, E. G., Aoki, T. T., Unger, R.H., Soeldner, J.S., Cahill, G.I., Jr.: Glucagon levels and metabolic effects in fasting man. J. clin. Invest 49, 2256-2270 (1970)

42. Unger, R. H.: Glucagon physiology and pathophysiology. New Engl. J. Med. 285, 443-449 (1971)

Prof. I. Bihler

Dept. of Pharmacology and Therapeutics

Faculty of Medicine

University of Manitoba

770 Bannatyne Avenue

Winnipeg, Manitoba R3E OW3

Canada 